\title{
A nomogram for Antenatal Estimation of Cephalopelvic Disproportion in Primiparous Women based on MRI Measurements
}

\author{
Cheng Chen ${ }^{1}$, Mengmeng Yang ${ }^{2}$, Weizeng Zheng ${ }^{3}$, Yuan Chen ${ }^{4}$, tian dong ${ }^{5}, \mathrm{Min} \mathrm{Lv}^{1}$, \\ Xiaofu Yang ${ }^{6}$, Fangfang Xi ${ }^{6}$, Ying Jiang ${ }^{1}$, Xia Ying ${ }^{6}$, Wen $\mathrm{Li}^{1}$, Jian $\mathrm{Xu}^{7}$, baihui zhao ${ }^{5}$, and \\ Qiong Luo $^{6}$
}

${ }^{1}$ Affiliation not available

${ }^{2}$ Women's Hospital School of Medicine Zhejiang University

${ }^{3}$ Women's Hospital, Zhejiang University School of Medicine

${ }^{4}$ Zhejiang University School of Medicine Women's Hospital

${ }^{5}$ Zhejiang University

${ }^{6}$ Womens Hospital, Zhejiang University School of Medicine

7 The Fourth Affiliated Hospital, Zhejiang University School of Medicine

January 6, 2022

\begin{abstract}
Objective: To develop and validate a predictive model assessing the risk of cesarean delivery in primiparous women based on the findings of magnetic resonance imaging (MRI) studies. Design: Observational study Setting: University teaching hospital. Population: 168 primiparous women with clinical findings suggestive of cephalopelvic disproportion. Methods: All women underwent MRI measurements prior to the onset of labor. A nomogram model to predict the risk of cesarean delivery was proposed based on the MRI data. The discrimination of the model was calculated by the area under the receiver operating characteristic curve (AUC) and calibration was assessed by calibration plots. The decision curve analysis was applied to evaluate the net clinical benefit. Main Outcome Measures: Cesarean delivery. Results: A total of 88 (58.7\%) women achieved vaginal delivery, and $62(41.3 \%)$ required cesarean section caused by obstructed labor. In multivariable modeling, the maternal body mass index before delivery, induction of labor, bilateral femoral head distance, obstetric conjugate, fetal head circumference and fetal abdominal circumference were significantly associated with the likelihood of cesarean delivery. The discrimination calculated as the AUC was 0.845 (95\% CI: 0.783-0.908; P < 0.001). The sensitivity and specificity of the nomogram model were 0.918 and 0.629 , respectively. The model demonstrated satisfactory calibration. Moreover, the decision curve analysis proved the superior net benefit of the model compared with each factor included. Conclusion: Our study provides a nomogram model that can accurately identify primiparous women at risk of cesarean delivery caused by cephalopelvic disproportion based on the MRI measurements.
\end{abstract} A nomogram for Antenatal Estimation of Cephalopelvic Disproportion inPrimiparous Women
based on MRI Measurements

\section{Authors}

Cheng Chen, M.D. ${ }^{1 *}$, Mengmeng Yang, M.D. ${ }^{1 *}$, Weizeng Zheng, M.D.+, Yuan Chen, M.D.*, Tian Dong, M.D.*, Min Lv, M.D.*, Xiaofu Yang, M.D.*, Fangfang Xi, M.D.*, Ying Jiang, M.D.*, Xia Ying, Ph.D. *, Wen Li, M.D.*, Jian Xu Ph.D.§*, Baihui Zhao, M.D.§*, Qiong Luo, Ph.D.*

\section{Affiliations}


*Department of Obstetrics, Women's Hospital, Zhejiang University School of Medicine, Hangzhou, China

+Department of Radiology, Women's Hospital, Zhejiang University School of Medicine, Hangzhou, China

SSDepartment of Obstetrics, the Fourth Affiliated Hospital, Zhejiang University School of Medicine, Yiwu, China

1Authors contributed equally to this study.

\section{Correspondence to:}

Pro Q. Luo, Department of Obstetrics, Women's Hospital, Zhejiang University School of Medicine, Hangzhou, 310006, China; tel: E-mail: luoq@zju.edu.cn)

Dr. B. Zhao, Department of Obstetrics, the Fourth Affiliated Hospital, Zhejiang University School of Medicine, Yiwu, 322000, China; (E-mail:zhaobh@zju.edu.cn)

Pro J. Xu, Department of Reproductive Center, the Fourth Affiliated Hospital, Zhejiang University School of Medicine, Yiwu, 322000, China; (E-mail: xuj@zju.edu.cn)

\section{Running title:}

A nomogram for predicting cesarean delivery

\section{Abstract}

Objective: To develop and validate a predictive model assessing the risk of cesarean delivery in primiparous women based on the findings of magnetic resonance imaging (MRI) studies.

Design: Observational study

Setting: University teaching hospital.

Population: 168 primiparous women with clinical findings suggestive of cephalopelvic disproportion.

Methods: All women underwent MRI measurements prior to the onset of labor. A nomogram model to predict the risk of cesarean delivery was proposed based on the MRI data. The discrimination of the model was calculated by the area under the receiver operating characteristic curve (AUC) and calibration was assessed by calibration plots. The decision curve analysis was applied to evaluate the net clinical benefit.

Main Outcome Measures: Cesarean delivery.

Results: A total of $88(58.7 \%)$ women achieved vaginal delivery, and $62(41.3 \%)$ required cesarean section caused by obstructed labor. In multivariable modeling, the maternal body mass index before delivery, induction of labor, bilateral femoral head distance, obstetric conjugate, fetal head circumference and fetal abdominal circumference were significantly associated with the likelihood of cesarean delivery. The discrimination calculated as the AUC was 0.845 (95\% CI: 0.783-0.908; $P<0.001)$. The sensitivity and specificity of the nomogram model were 0.918 and 0.629 , respectively. The model demonstrated satisfactory calibration. Moreover, the decision curve analysis proved the superior net benefit of the model compared with each factor included.

Conclusion: Our study provides a nomogram model that can accurately identify primiparous women at risk of cesarean delivery caused by cephalopelvic disproportion based on the MRI measurements.

Keywords : Nomograms, Pelvimetry, Cephalopelvic disproportion, Cesarean section

\section{Tweetable abstract}

A model may guide the clinician in making appropriate management decisions for primiparous women with clinical suspected cephalopelvic disproportion.

\section{INTRODUCTION}


Cephalopelvic disproportion (CPD) is a mismatch between the maternal pelvis and the fetus, which is the most common cause of obstructed labor ${ }^{1}$. Dystocia and subsequent emergency cesarean section are associated with maternal and neonatal morbidity, including uterine rupture, postpartum hemorrhage, chorioamnionitis, neonatal birth injuries, and even mortality ${ }^{2}, 3$. On the other hand, the over-diagnosis of CPD is one of the main reasons for a continuous increase in cesarean deliveries ${ }^{4}$. If obstetricians and midwives could identify patients at high risk for cesarean delivery, it might avoid increased complications by offering a scheduled surgery, while those at low risk could be encouraged to pursue vaginal delivery.

Clinical pelvimetry to estimate various pelvic dimensions using systematic manual examinations of specific bony landmarks has been considered poor accurate and unsatisfactory interobserver agreement ${ }^{5}$. Hence, radiological pelvimetry measurements using $\mathrm{X}$-ray ${ }^{6}$, computed tomography ${ }^{7}$ and magnetic resonance imaging $(\mathrm{MRI})^{8}$ have been introduced to antenatal assess the relationship between the maternal pelvis and fetus in order to choose proper delivery methods. MRI pelvimetry is superior to other radiological techniques, including X-ray and computed tomography, since it can provide an accurate evaluation of pelvic dimensions while imaging soft tissue structures and fetus without radiation exposure ${ }^{9,}{ }^{10}$. The pelvimetric errors of MRI are approximate $1 \%$ versus $10 \%$ for conventional X-ray measurements ${ }^{8}$. Although the MRI seemed promising to evaluate the maternal pelvic capacity and fetal size, to our knowledge, yet there is still no literature that has focused on the model using MRI to predict the chance of successful vaginal delivery.

As such, the objective of our study was to prospectively assess the clinical and MRI features to develop and validate a risk prediction model for estimating CPD in primiparous women before the onset of labor.

\section{METHODS}

\section{Study design}

The prospective, blinded observational study was conducted between April 2020 and April 2021 at Women Hospital, Zhejiang University, school of medicine. Nulliparous women were eligible if they had a viable singleton cephalic presentation pregnancy at full term ([?] 37 completed weeks) with clinical suspicion of CPD (either short maternal stature, unengaged fetal presentation or suspected macrosomia). Exclusion criteria were congenital malformations, maternal contraindications to vaginal delivery and contraindications to the use of MRI such as severe claustrophobia and metal implants.

The enrolled women had spontaneous onset of labor or performed induction of labor. Failure to labor progress was defined according to national guidelines ${ }^{11}$. Cesarean delivery was recommended if failure to generate cervical change after at least 24 hours of oxytocin administration during the latent phase or the rate of cervical dilation $<1 \mathrm{~cm}$ in 4 hours when the cervix was $>6 \mathrm{~cm}$ dilated $^{11}$. The women were also excluded if they required cesarean deliveries with other indications, such as fetal distress, placental abruption and preeclampsia. Managing practitioners were blinded to the results of MRI findings. Vaginal delivery included spontaneous and assisted operative deliveries using forceps.

The study was approved by the local ethics and research committees (IRB-20200044-R on April 04, 2020). In addition, written consent was obtained from all women who agreed to participate.

\section{MRI pelvimetry and fetalbiometry}

MRI measurements were performed within fourteen days before labor. All antenatal MRI images were obtained using a 1.5-T unit system (GE Signal HDxt; GE Healthcare, USA) and an eight-element phasedarray body coil. The enrolled women were placed in a supine position without sedation. After a localizing gradient echo sequence, the imaging protocol consisted of axial T1-weighted fast spin-echo and sagittal T1 fast spin-echo sequences (repetition time $520 \mathrm{~ms}$, echo time $7.8 \mathrm{~ms}$, $5 \mathrm{~mm}$ slice thickness, $0.5 \mathrm{~mm}$ gap, a field of view 36-40 cm). The scan extended down to the level of the lower margin of the pubic symphysis. Parameters were measured using the institution's picture archiving and communication system (Zhejiang Greenlander I.T. Co., Ltd., Hangzhou, China).

On transverse sections, bilateral femoral head distance, interspinous distance, intertuberous distance and 
subpubic angle were visualized and measured (Figure S1). The midsagittal section measurements included the following parameters: obstetric conjugate, pelvic width, sacral outlet diameter, outlet diameter of the pelvis, sacrum length and pelvic inclination (Figure S2). Fetal biometry, including fetal biparietal diameter, head circumstance and abdominal circumstance, were obtained from the 4-mm acquisition. All parameters were measured independently by two radiologists with at least five years of experience in gynecological MRI for inter-observer and intra-observer reliability.

Maternal characteristics including age, gestational age at delivery, height, weight, weight gain during pregnancy, induced or spontaneous labor and the final mode of delivery were recorded. Data on the neonatal outcomes were also collected: sex, birth weight, Apgar scores after 1 and 5 minutes and the admission of the newborn to the neonatal unit. Gestational age was determined by the first day of the last menstrual period and confirmed by the first-trimester ultrasound measurement of crown-rump length ${ }^{12}$. Body mass index (BMI) was calculated according to the standard formula.

\section{Statistical analysis}

The baseline characteristics were described using means (standard deviations [SDs]) for continuous variables and numbers (proportions) for categorical variables. The candidate variables associated with the risk of cesarean delivery were selected a priori based on the clinical feature, common sense and predictors assessed in the previously published literature ${ }^{13-15}$. Univariable logistics regression analyses were performed to estimate odds ratios (OR) and 95\% confidence intervals (CIs). We excluded maternal height and fetal biparietal diameter as the candidate predictors because of the multicollinearity with other variables. A backward stepwise elimination approach was applied to select independent variables for the multivariable logistics regression model with the Akaike Information Criterion ${ }^{16}$.

Nomograms were constructed by selected variables to predict the probability of cesarean section using statistical software (rms in R; http:// www.r-project.org). For model performance, we assessed the discrimination (the ability to differentiate between the prediction and outcome) and calibration (the discrepancy between predicted and observed outcomes). To quantify the model discrimination, we calculated the concordance (C-) index as the area under the receiver operating characteristic curve (AUC). The calibration was evaluated by calibration plots, accompanied by the Hosmer-Lemeshow goodness-of-fit test. The model internal validation was accessed by bootstrapped resampling to quantify overoptimism ${ }^{17}$. The optimal cutoff value was calculated by maximizing the Youden index using receiver operating characteristic curve (ROC) analysis (i.e., sensitivity + specificity - 1), and then the sensitivity and specificity were estimated. Finally, decision curve analyses were also applied to evaluate the net benefit of the prediction model (rmda in R; http:// www.r-project.org $)^{18}$. All tests were 2 -sided, $P$ value less than 0.05 was considered statistically significant. The analyses were performed using $\mathrm{R}$ statistical software, version 4.0.3.

\section{RESULTS}

\section{Study population}

A total of 208 nulliparous women were recruited into our study. We excluded 27 women who decided elective cesarean delivery for the following reasons: changed their minds and rejected attempting to a vaginal delivery after an MRI examination $(\mathrm{n}=25)$, pre-eclampsia $(\mathrm{n}=2)$. Of the eligible 181 women, twenty-nine were excluded for immediate urgency situation of fetal compromise, one was excluded for incomplete data and one withdrew her consent (Figure S1). Table 1 illustrates the baseline clinical characteristics, MRI measurements for the study population. Of the remaining 150 women for analysis, the mean age was 30 years and the mean height was $158 \mathrm{~cm}$. Sixty-two women required cesarean delivery (41\%), while eighty-eight women $(59 \%)$ underwent vaginal delivery: among these, 12 women (8\%) were performed assisted operative deliveries using forceps and $76(51 \%)$ achieved spontaneous vaginal delivery.

\section{Candidate predictors of cesarean delivery}

Univariable analyses revealed the following variables were significantly associated with the probability of caesarean delivery: maternal height, BMI before labor, induction of labor, bilateral femoral head distance, 
interspinous diameter, obstetric conjugate, subpubic angle, fetal biparietal diameter, fetal head circumference and fetal abdominal circumference (Table 2). Subsequent backward stepwise multivariable logistics analysis identified maternal BMI before labor (OR, 1.307; 95\% CI, 1.109-1.541; $P=0.001$ ), induction (OR, 2.777; 95\% CI, 1.190-6.478; $P=0.018$ ), bilateral femoral head distance (OR, 0.898; 95\% CI, 0.838-0.963; $P=$ 0.003 ), obstetric conjugate (OR, 0.951; 95\% CI, 0.907-0.997; $P=0.038)$, fetal head circumference (OR, 1.786; 95\% CI, 1.152-2.770; $P=0.01$ ) and fetal abdominal circumference (OR, 1.685; 95\% CI, 1.221-2.325; $P=0.001)$ as independent predictors for the risk of cesarean delivery (Table 3).

\section{Model Performance}

Nomograms derived from the multivariable model to predict the probability of cesarean delivery were shown in Figure 1. The sum of the assigned points for each variable in the nomograms predicted the overall risk. The discrimination of this prediction model was evaluated using the AUC $(0.85,95 \%$ CI: $0.79-0.91 ; P<$ 0.001) (Figure 2a). The calibration plots showed good agreement between prediction and observation with a calibration slope of 1.01 (Figure $2 \mathrm{~b}$ ). Additionally, the Hosmer-Lemeshow test yielded a nonsignificant statistic $(P=0.472)$, which indicated a reasonable model fit.

\section{Clinical significance}

The sensitivity and specificity of the prediction model were 0.90 and 0.66 , respectively. The decision curve analyses to compare the performance of the nomograms with the univariable models representing individual variables selected by the nomograms are provided in Figure 3. The nomogram model obtained a more significant net benefit than the models of individual predictors.

\section{DISCUSSION}

\section{Main Findings}

In this prospective cohort study, we develop and validate a nomogram model to predict the probability of cesarean delivery for nulliparous women with suspected cephalopelvic disproportion based on six parameters: maternal BMI before labor, induction of labor (spontaneous / induced), bilateral femoral head distance, obstetric conjugate, fetal head circumference and fetal abdominal circumference. It is the first time combining maternal characteristics and MRI parameters as a practical tool to evaluate individual CPD risk in the nulliparous woman at term.

\section{Strengths and limitations}

Our study has several limitations. The main drawback of this model is the comparatively high cost of MRI examination. Generally, the current cost of MR imaging is approximately 3- to 4-fold the cost of ultrasonography. However, we simplified the acquisition parameters of the MRI examination for this model, which should reduce the cost of MRI for this specific population with suspected CPD. Hopefully, with the simplification of the MRI examination, the benefit of this method would outweigh the cost arguments. In addition, our study population included only Chinese women, and our results might not be representative of other ethnic populations. Furthermore, we only performed internal validation using bootstrap resampling, and further external validation should be required from other populations. Despite these limitations, we constructed a nomogram model to predict the risk of cesarean delivery with good accuracy; to our knowledge, it potentially was the first time.

\section{Interpretation (in light of other evidence)}

Antenatal estimation of the relationship between the maternal pelvis and fetal size is essential to evaluate obstetrical prognosis. Various pelvic diameters have been proposed to assess the risk of unplanned cesarean delivery. Several studies reported a significant positive correlation between obstetric conjugate and vaginal delivery ${ }^{15,19}$. Besides, Joyce et al. ${ }^{20}$ asserted the minimum obstetric conjugate of $10 \mathrm{~cm}$ that required a fetus of $3400 \mathrm{~g}$ to pass through the birth canal. Similarly, our final model incorporated the obstetric conjugate as a significant predictive factor for dystocia. Another significant variable is the interspinous diameter, which is considered as the parameter representing mid-pelvis. Our study showed that the interspinous diameter 
was significantly associated with the cesarean delivery group by univariable analysis, which agrees with the previous report ${ }^{15,21}$. However, the final multivariate model eliminated the interspinous diameter as an independent factor to predict the overall cesarean risk for dystocia. Moreover, we identified the bilateral femoral head diameter as a predictive factor in both univariable and multivariate analysis, which can be a novel parameter measuring the transverse distance of the mid-pelvis. The mid-pelvis was considered as an elemental plane of the birth canal, through which the fetus' head usually passes in sagittal orientation. Therefore, the bilateral femoral head distance, which is located in the central of the maternal pelvis in anatomical structure, can be a reliable parameter to assess the capacity of the mid-pelvis.

Because unplanned cesarean delivery is associated with a greater risk of maternal morbidity and surgical complications than scheduled cesarean delivery and vaginal delivery ${ }^{22}$, it would be clinically beneficial to predict the labor outcome in women with suspected CPD. There have been previous efforts to provide predictive models for quantifying CPD. Morgan et al. originally proposed the fetal pelvic index ${ }^{6}$, which combined fetal head and abdominal circumferences with maternal pelvic inlet and mid pelvis circumferences to identify CPD. However, it has been criticized for the clinically useful as a poor overall prediction value $\mathrm{e}^{23,24}$. Burke et al. developed a nomogram based on five parameters (maternal age, height, BMI, fetal abdominal circumference and fetal head circumference) to assess the risk of primary cesarean delivery with limited predictive accuracy $(\mathrm{AUC}=0.69)^{25}$. Nevertheless, previous models were either limited diagnostic accuracy ${ }^{25,26}$ or too complex for routine clinical practice $\mathrm{e}^{27,28}$. Additionally, some prediction tools required intrapartum factors that counseling about likely outcomes cannot be made until labor occurs ${ }^{29}$. Our nomogram model based on antepartum factors may be a clinically beneficial and accurate tool for predicting cesarean delivery risk in nulliparous women.

Previous studies have demonstrated that MRI is a reliable and accurate method to evaluate labor outcomes irrespective of the experience. A randomized trial conducted by Van Loon et. al. ${ }^{30}$ asserted that MRI pelvic measurements allowed the better choice of delivery modes with a significantly lower emergency cesarean section rate of breech presentation at term. Some researchers attempted to identify if MRI pelvimetry could predict the success of vaginal delivery in women with previous cesarean sections ${ }^{31}$. As the study comprised only 16 patients, we considered the results should be supported by larger ones. Sporri et. al. ${ }^{21}$ confirmed the significant association between MRI pelvimetry and CPD in 42 women, especially the parameters of the interspinous dimension and obstetric conjugate. However, the inclusion criteria were unrepresentative and MRI pelvimetry was performed postpartum. Previous attempts have shown that several MRI pelvimetric parameters are associated with $\mathrm{CPD}$, such as anterior-posterior mid-pelvis diameter, interspinous diameter, and mid-pelvis capacity ${ }^{15}, 32,33$. Another application of MRI is to estimate fetal weight; compared with ultrasound biometry, it is more accurate ${ }^{34}$. To date, there has not been an established method for accessing the effects of multiple risk factors incorporated MRI measurements for determining the probability of primary cesarean delivery in an individual woman. Our study expands the findings of previous investigations to develop a nomogram model based on pelvic dimensions and fetal biometry measured by MRI to predict successful vaginal delivery.

\section{CONCLUSION}

In conclusion, we conducted a nomogram by combining six prepartum risk factors to predict the likelihood of cesarean delivery in primiparous women at term. This nomogram provides good discrimination and calibration. In addition, the decision curve analysis shows net beneficial in conventionally clinical practice.

\section{Disclosure of Interests}

The authors report no conflict of interest.

\section{Contribution to Authorship}

Concept and design: CC, JX, BHZ and QL.

Data acquisition: MMY, WZZ, YC, FFX, YJ and TD. 
Data cleaning and management: ML, XY, WL,

Statistical analysis: CC, ML, XFY and XY

Drafting of manuscript: CC, BHZ and QL.

Review of manuscript: all authors.

\section{Details of Ethics Approval}

The study protocol was approved by the Ethical Committee of Women Hospital, Zhejiang University, School of Medicine in Hangzhou (IRB-20200044-R on April 04, 2020) and has been carried out according to the declaration of Helsinki.

\section{Funding:}

This work was supported by Natural Science Foundation of Zhejiang Province (LQ20H040008), and Scientific Research Foundation of the National Health Commission (WKJ-ZJ-2126).

\section{Acknowledgments:}

No acknowledgments.

\section{References}

1. El-Hamamy E, Arulkumaran S. Poor progress of labour. Current Obstetrics \& Gynaecology. 2005 2005/02/01/;15(1):1-8.

2. Rosenbloom JI, Stout MJ, Tuuli MG, Woolfolk CL, Lopez JD, Macones GA, et al. New labor management guidelines and changes in cesarean delivery patterns. Am J Obstet Gynecol. 2017 2017/12/01/;217(6):689.e1.e8.

3. Caughey AB, Cahill AG, Guise J-M, Rouse DJ. Safe prevention of the primary cesarean delivery. Am J Obstet Gynecol. 2014 2014/03/01/;210(3):179-93.

4. Charoenboon C, Srisupundit K, Tongsong T. Rise in cesarean section rate over a 20-year period in a public sector hospital in northern Thailand. Arch Gynecol Obstet. 2013 Jan;287(1):47-52.

5. Maharaj D. Assessing cephalopelvic disproportion: back to the basics. Obstetrical \& gynecological survey. 2010 Jun;65(6):387-95.

6. Morgan MA, Thurnau GR, Fishburne JI, Jr. The fetal-pelvic index as an indicator of fetal-pelvic disproportion: a preliminary report. Am J Obstet Gynecol. 1986 Sep;155(3):608-13.

7. Lenhard M, Johnson T, Weckbach S, Nikolaou K, Friese K, Hasbargen U. Three-dimensional pelvimetry by computed tomography. La radiologia medica. 2009 2009/06/23;114(5):827.

8. Stark DD, McCarthy SM, Filly RA, Parer JT, Hricak H, Callen PW. Pelvimetry by magnetic resonance imaging. AJR American journal of roentgenology. 1985 May;144(5):947-50.

9. Prayer D, Malinger G, Brugger PC, Cassady C, De Catte L, De Keersmaecker B, et al. ISUOG Practice Guidelines: performance of fetal magnetic resonance imaging. Ultrasound Obstet Gynecol. 2017 May;49(5):671-80.

10. Malin GL, Bugg GJ, Takwoingi Y, Thornton JG, Jones NW. Antenatal magnetic resonance imaging versus ultrasound for predicting neonatal macrosomia: a systematic review and meta-analysis. BJOG. 2016 Jan;123(1):77-88.

11. Spong CY, Berghella V, Wenstrom KD, Mercer BM, Saade GR. Preventing the First Cesarean Delivery: Summary of a Joint: Eunice Kennedy Shriver: National Institute of Child Health and Human Development, Society for Maternal-Fetal Medicine, and American College of Obstetricians and Gynecologists Workshop. Obstetrics \& Gynecology. 2012;120(5). 
12. Napolitano R, Dhami J, Ohuma EO, Ioannou C, Conde-Agudelo A, Kennedy SH, et al. Pregnancy dating by fetal crown-rump length: a systematic review of charts. BJOG. 2014 Apr;121(5):556-65.

13. Heffner LJ, Elkin E, Fretts RC. Impact of labor induction, gestational age, and maternal age on cesarean delivery rates. Obstetrics \& Gynecology. 2003 2003/08/01/;102(2):287-93.

14. Rossi RM, Requarth E, Warshak CR, Dufendach KR, Hall ES, DeFranco EA. Risk Calculator to Predict Cesarean Delivery Among Women Undergoing Induction of Labor. Obstet Gynecol. 2020 Mar;135(3):559-68.

15. Zaretsky MV, Alexander JM, McIntire DD, Hatab MR, Twickler DM, Leveno KJ. Magnetic resonance imaging pelvimetry and the prediction of labor dystocia. Obstet Gynecol. 2005 Nov;106(5 Pt 1):919-26.

16. Kim Y, Margonis GA, Prescott JD, Tran TB, Postlewait LM, Maithel SK, et al. Nomograms to Predict Recurrence-Free and Overall Survival After Curative Resection of Adrenocortical Carcinoma. JAMA surgery. 2016 Apr;151(4):365-73.

17. Harrell FE, Jr., Lee KL, Mark DB. Multivariable prognostic models: issues in developing models, evaluating assumptions and adequacy, and measuring and reducing errors. Statistics in medicine. 1996 Feb $28 ; 15(4): 361-87$.

18. Vickers AJ, Cronin AM. Traditional statistical methods for evaluating prediction models are uninformative as to clinical value: towards a decision analytic framework. Seminars in oncology. 2010 Feb;37(1):31-8.

19. Franz M, von Bismarck A, Delius M, Ertl-Wagner B, Deppe C, Mahner S, et al. MR pelvimetry: prognosis for successful vaginal delivery in patients with suspected fetopelvic disproportion or breech presentation at term. Arch Gynecol Obstet. 2017 Feb;295(2):351-9.

20. Joyce DN, Giwa-Osagie F, Stevenson GW. Role of pelvimetry in active management of labour. British medical journal. 1975 Nov 29;4(5995):505-7.

21. Sporri S, Hanggi W, Braghetti A, Vock P, Schneider H. Pelvimetry by magnetic resonance imaging as a diagnostic tool to evaluate dystocia. Obstet Gynecol. 1997 Jun;89(6):902-8.

22. Lilford RJ, van Coeverden de Groot HA, Moore PJ, Bingham P. The relative risks of caesarean section (intrapartum and elective) and vaginal delivery: a detailed analysis to exclude the effects of medical disorders and other acute pre-existing physiological disturbances. British journal of obstetrics and gynaecology. 1990 Oct;97(10):883-92.

23. Macones GA, Chang JJ, Stamilio DM, Odibo AO, Wang J, Cahill AG. Prediction of cesarean delivery using the fetal-pelvic index. Am J Obstet Gynecol. 2013 Nov;209(5):431.e1-8.

24. Ferguson JE, 2nd, Newberry YG, DeAngelis GA, Finnerty JJ, Agarwal S, Turkheimer E. The fetalpelvic index has minimal utility in predicting fetal-pelvic disproportion. Am J Obstet Gynecol. 1998 Nov;179(5):1186-92.

25. Burke N, Burke G, Breathnach F, McAuliffe F, Morrison J, Turner M, et al. Prediction of cesarean delivery in the term nulliparous woman: results from the prospective, multicenter Genesis study. American journal of obstetrics and gynecology. 2017;216(6):598.e1-.e11.

26. Smith G, Dellens M, White I, Pell J. Combined logistic and Bayesian modeling of cesarean section risk. American journal of obstetrics gynecology. 2004;191(6):2029-34.

27. Al Housseini A, Newman T, Cox A, Devoe LD. Prediction of risk for cesarean delivery in term nulliparas: a comparison of neural network and multiple logistic regression models. Am J Obstet Gynecol. 2009 2009/07/01/;201(1):113.e1-.e6.

28. Sims CJ, Meyn L, Caruana R, Rao RB, Mitchell T, Krohn M. Predicting cesarean delivery with decision tree models. Am J Obstet Gynecol. 2000 2000/11/01/;183(5):1198-206. 
29. Costantine MM, Fox K, Byers BD, Mateus J, Ghulmiyyah LM, Blackwell S, et al. Validation of the prediction model for success of vaginal birth after cesarean delivery. Obstet Gynecol. 2009 Nov;114(5):102933.

30. van Loon AJ, Mantingh A, Serlier EK, Kroon G, Mooyaart EL, Huisjes HJ. Randomised controlled trial of magnetic-resonance pelvimetry in breech presentation at term. Lancet. 1997 Dec 20-27;350(9094):1799804.

31. Fox L, Huerta-Enochian G, Hamlin J, Katz V, gynecology. The magnetic resonance imaging-based fetal-pelvic index: a pilot study in the community hospital. American Journal of Obstetrics \& Gynecology. 2004;190(6):1679-85; discussion 85-8.

32. Mengert WF. Estimation of pelvic capacity. Journal of the American Medical Association. 1948 Sep 18;138(3):169-74.

33. Li YG, Chen CL, Liao KD, Yu YH, Xu YK, Wang Y, et al. Study on the cephalopelvic relationship with cephalic presentation in nulliparous full-term Chinese pregnant women by MRI with three-dimensional reconstruction. Arch Gynecol Obstet. 2018 Aug;298(2):433-41.

34. Zaretsky M, Reichel T, McIntire D, Twickler D, gynecology. Comparison of magnetic resonance imaging to ultrasound in the estimation of birth weight at term. American Journal of Obstetrics \& Gynecology. 2003;189(4):1017-20.
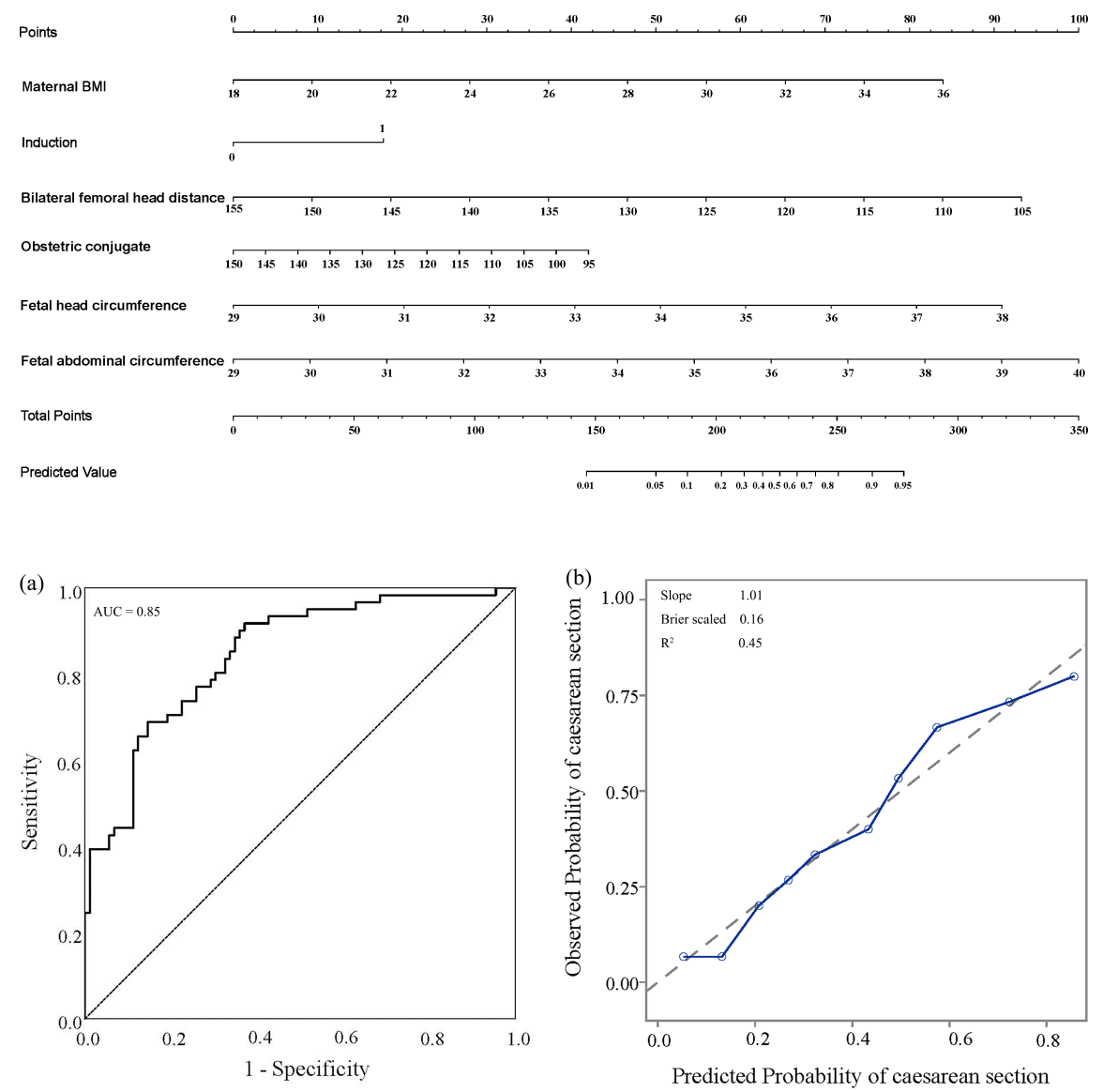


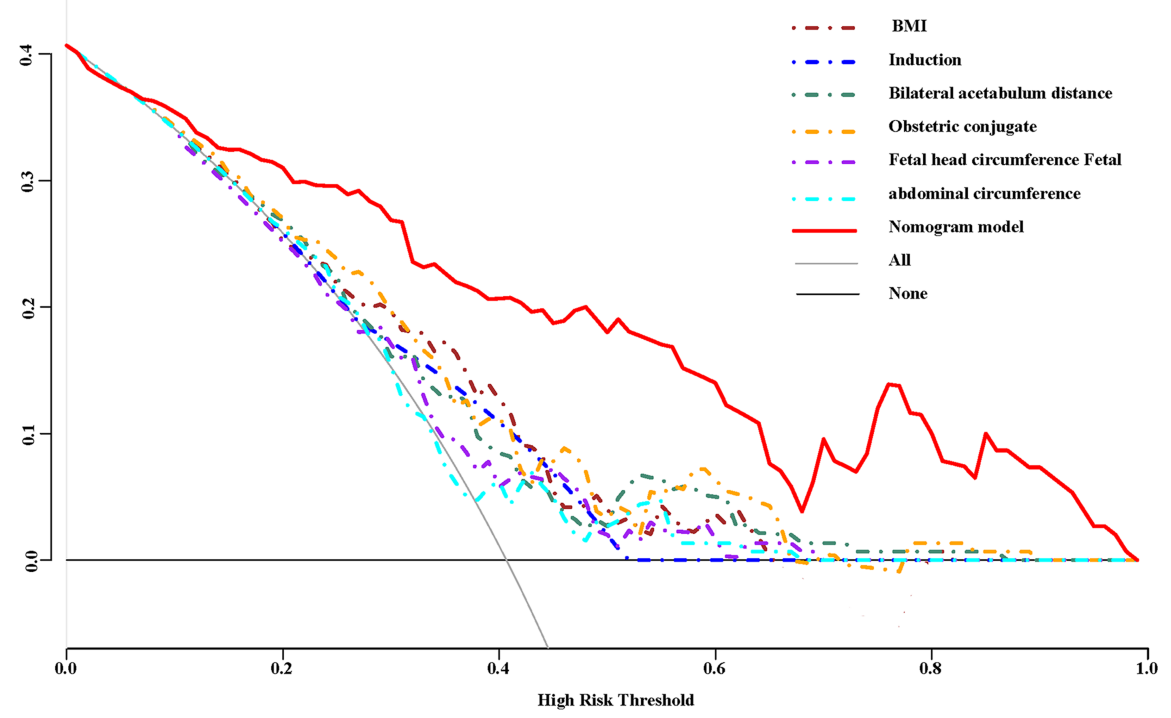

